\title{
Percorsi diagnostico-terapeutici nella gestio- ne dei pazienti affetti da polmonite acquisita in comunità
}

Mario Eandi*

PERCORSI

\begin{abstract}
Community-Acquired Pneumonia (CAP) is one of the major cause of death due to infectious diseases in developed countries. In Italy, about $18 \%$ of the patients with CAP are hospitalized, accounting for an annual health-care cost of more than 1.000 billion Lire. Overall, CAP represents an heavy burden to the society and the National Health-Care System.

The managed-care of patients with CAP should be committed to find a balance between the individual health-care needs and the more general claims of a fair utilization and an overall efficiency of the healthcare system.

The comprehensive strategy of care should consider many decision nodes and the outcomes related with each different options. The diagnostic procedures (physical examination, chest radiography, microbiology, laboratory) should be oriented to obtain a robust differential diagnosis and to estimate the risk of mortality. Prediction rule are now available that help physicians to make more rational decisions about hospitalization for patients with pneumonia.

The selection of antibiotic in the absence of an etiologic diagnosis is based on the epidemiological settings, the severity of the illness and other clinical conditions of the patients. Non concordant therapeutic recommendations are provided by the published guidelines.

Decisions to be taken during the follow-up concern the switch therapy and the early discharge options, as soon as the patient stabilizes, or the admission to ICU in case of worsening. The length of hospital stay depends on the time to stability and the risk of mortality. However, socio-cultural factors and availability of outpatient care programs could facilitate the early discharge of patients and help to decrease the health-care costs of CAP patients.

The results of the present review can be used as a background to implement a general decision model suitable to performe pharmacoeconomic analysis of the CAP therapy.
\end{abstract}

Farmeconomia e percorsi terapeutici 2000; 1 (1): 35-52

\section{INTRODUZIONE}

L'analisi farmacoeconomica richiede che vengano individuate tutte le alternative terapeutiche significative di una data malattia o sindrome clinica. Nella maggior parte dei casi la decisione fondamentale riguarda la scelta tra due o più farmaci, oppure la scelta tra una terapia farmacologica e altre terapie non farmacologiche, compresa l'opzione di non attuare alcun trattamento.

In realtà la scelta di una terapia è quasi sempre condizionata da un precedente iter diagnostico e, d'altra parte, la conduzione della terapia richiede sempre un'attenta e precisa osservazione del paziente per monitorare l'andamento clinico, evidenziare i segni di miglioramento o peggioramento al fine di poter prendere tempestivamente le decisioni di riconsiderare eventualmente la diagnosi e di modificare, se necessario, la terapia.
Inoltre, le condizioni cliniche del paziente, il suo contesto socio-culturale, l'organizzazione dell'assistenza sanitaria nella zona in cui vive, così come le caratteristiche farmacologiche e terapeutiche dei farmaci disponibili, sono fattori che determinano le possibili alternative strategiche di gestione complessiva di un paziente.

L'analisi farmacoeconomica, pertanto, può svolgere completamente la sua funzione di supporto ai decisori istituzionali, rappresentati dalla Società e dal Sistema Sanitario Nazionale (SSN), qualora il processo decisionale che riguarda la scelta del trattamento farmacologico venga inserito in un più complessivo percorso diagnostico-terapeutico del paziente affetto da una data malattia o sindrome clinica.

La polmonite acquisita in comunità (CAP) può essere presa come esempio paradigmatico per illustrare l'importanza che assume, per
* Dipartimento di Anatomia, Farmacologia e Medicina Legale, Sezione di Farmacologia, Università di Torino. 
l'analisi farmacoeconomica, l'identificazione dei diversi possibili percorsi diagnosticoterapeutici attuati nella gestione dei singoli pazienti.

La polmonite acquisita in comunità, nonostante i progressi indotti dall'introduzione degli antibiotici, continua a essere un'infezione a incidenza annuale relativamente frequente che comporta una elevata morbilità e mortalità e costi sanitari e sociali molto pesanti .

Indagini effettuate negli Stati Uniti negli anni ' 80 avevano evidenziato che la CAP veniva diagnosticata in circa 4 milioni di adulti ogni anno e che circa 600.000 di questi venivano ricoverati (1). Il costo aggregato annuale della sola ospedalizzazione per CAP era stato allora stimato in circa 4 miliardi di dollari (2, 3). Nel 1994 il numero di ricoveri per polmonite negli Stati Uniti è stato di circa 1 milione per un costo di oltre 6 miliardi di dollari, pari a oltre 13 mila miliardi di lire attuali (4-6).

Alcune stime condotte anche in Europa indicano in circa un ricovero ogni mille abitanti l'incidenza annuale di ricoveri ospedalieri per CAP (7). In Italia negli anni 1993-1995 il numero annuale di ricoveri per infezioni delle basse vie respiratorie è stato stimato in circa 450.000, dei quali circa 200.000 erano rappresentati da polmoniti acquisite in comunità. Complessivamente per la CAP sono state consumate ogni anno oltre 2,5 milioni di giornate di degenza e il costo annuale sostenuto dal SSN per pagare i ricoveri è stato stimato, utilizzando il valore delle tariffe DRG pertinenti, in oltre 1.000 miliardi di Lire (8).

La percentuale di ricoveri per CAP varia molto da una nazione all'altra e da un'area geografica all'altra $(9,10)$. In Italia il ricovero ospedaliero per infezioni delle basse vie respiratorie avviene in circa il 2,8\% di casi: si tratta di una frequenza nettamente inferiore alla media europea $(4,5 \%)$, a quella francese $(5,1 \%)$ e a quella del Regno Unito (9\%) (10).

La diagnosi di sospetta polmonite è una delle motivazioni più frequenti che induce al ricovero in ospedale: i soggetti con sospetta polmonite hanno una probabilità su sei di essere ricoverati e tale probabilità aumenta nei soggetti anziani e nei soggetti che presentano condizioni cliniche gravi.

La variabilità delle percentuali di ricovero da zona a zona indica, tuttavia, che il medico non ha criteri uniformi, robusti e consolidati per decidere: egli spesso si basa sulla sua impressione soggettiva, talvolta tende a sovrastimare il rischio di morte del paziente affetto da polmonite e spesso ricovera pazienti a basso rischio, trattabili a domicilio $(6,11,12)$.

Nonostante tutti i pazienti affetti da polmonite siano trattati con antibiotici, essi, in termini prognostici, continuano ad avere una mortalità significativa che oscilla da circa il 5\% per i pazienti ospedalizzati o ambulatoriali fino al $37 \%$ nei pazienti che vengono ricoverati in terapia intensiva (13). Una recente indagine ha evidenziato che in Italia la mortalità associata alla CAP è almeno tre volte più elevata nei soggetti compresi tra i 55 ed i 74 anni ripetto ai giovani (14). Analogamente, nel Regno Unito circa il 95\% delle morti per polmoniti si concentra tra i pazienti che hanno oltre 65 anni di età (15).
Tabella 1

Variabililitàdella gestione diagnostica e terapeutica del paziente affetto da infezione delle basse vie respiratorie (LRTI) e in particolare da polmonite acquisita in comunità $(C A P)$ in cinque

Paesi europei. (Modificatada: Huchon GJ et al, 1996)(16)

\begin{tabular}{|l|c|c|c|c|c|c|}
\hline & Italia & Francia & Spagna & Germania & $\begin{array}{c}\text { Regno } \\
\text { Unito }\end{array}$ & Tutti \\
\hline $\begin{array}{l}\% \text { diagnosi CAP fatte } \\
\text { dal medico di Med } \\
\text { Generale }\end{array}$ & 18 & 17 & 19 & 18 & 18 & 18 \\
\hline $\begin{array}{l}\text { Richiesta di Rx, conta } \\
\text { GB, esame sputo } \\
\text { (\% su LRTI) }\end{array}$ & 24 & 21 & 36 & 43 & 18 & 29 \\
\hline $\begin{array}{l}\text { Ricoveri in ospedale } \\
\text { (\% su LRTI) }\end{array}$ & 3 & 5 & 3 & 3 & 9 & 5 \\
\hline $\begin{array}{l}\text { Pazienti senza } \\
\text { prescrizione antibiotica } \\
\text { (\% CAP) }\end{array}$ & 3 & 8 & 7 & 23 & 16 & 13 \\
\hline $\begin{array}{l}\text { Giorni terapia antibiotica } \\
\text { (media } \pm \text { SD) }\end{array}$ & $7,4 \pm 3,4$ & $9,2 \pm 3,0$ & $9,8 \pm 3,3$ & $9,3 \pm 4,8$ & $6,4 \pm 1,6$ & $8,4 \pm 3,6$ \\
\hline $\begin{array}{l}\text { Uso antibiotico per via } \\
\text { parenterale (\% CAP) }\end{array}$ & 71 & 17 & 15 & 6 & 0 & 21 \\
\hline \hline
\end{tabular}

$\mathrm{CAP}=$ Polmonite acquisita in comunità; LRTI = Infezione della basse vie respiratorie 
Questo alto rischio di mortalità evidenzia la necessità che il medico scelga attentamente come gestire il paziente affetto da polmonite e in particolare se trattarlo a domicilio o ricoverarlo in ospedale $(6,11)$.

Le CAP, come tutte le altre infezioni delle basse vie respiratorie, vengono generalmente gestite inizialmente dal medico di Medicina Generale. Egli deve conoscere come utilizzare al meglio l'esame clinico per identificare i pazienti a rischio di polmonite e poter arrivare a una diagnosi definitiva.

La corretta diagnosi clinica è anche una buona premessa per impostare un'appropriata terapia antibiotica che salvaguardi il paziente senza aumentare eccessivamente il rischio di insorgenza di resistenze batteriche. Il trattamento antibiotico iniziale delle CAP è per lo più empirico-ragionato, ma il medico, oggi, ha un ventaglio molto ampio di antibiotici tra i quali scegliere. Inoltre ha la possibilità di optare per la via orale piuttosto che per la via parenterale.

Le modalità di gestione di un paziente affetto da CAP variano sensibilmente nei diversi Paesi. Una recente indagine, condotta in cinque nazioni europee, ha evidenziato come la probabilità che il medico di Medicina Generale faccia diagnosi di CAP è sostanzialmente uguale (circa 18\%), mentre variano discretamente le percentuali di ricorso a indagini radiografiche, microbiologiche e di laboratorio e le percentuali di ricovero (vedi tabella 1). Trattare a domicilio o in ospedale condiziona l'accuratezza ed estensione della diagnosi, la modalità di trattamento e l'intensità delle visite e degli esami clinici.

Significative sono, inoltre, le differenze di comportamento nella prescrizione di antibio- tici, nella scelta della via di somministrazione e della durata del trattamento (16).

Negli ultimi anni diverse Società Scientifiche o singoli autori hanno pubblicato lineeguida per uniformare e razionalizzare la gestione del paziente affetto da CAP (17-24). Non abbiamo, al momento, indicazioni precise circa il livello di accettazione e di applicazione di tali linee-guida nella pratica quotidiana. Il presente lavoro non vuole essere un nuovo tentativo di proporre una linea-guida per la CAP, ma, partendo da quelle già pubblicate intende analizzare quali siano i nodi decisionali fondamentali che si incontrano nel percorso diagnostico terapeutico di un paziente affetto da CAP evidenziando in particolare quelle decisioni che sono rilevanti nel determinare un diverso consumo di risorse. Quest'analisi è preliminare per costruire un modello decisionale farmacoeconomico che presenteremo nella seconda parte del lavoro.

\section{NODI DECISIONALI \\ E LE CONSEGUENZE}

La figura 1 riassume i principali problemi che il medico deve affrontare nella gestione dei pazienti affetti da CAP o a rischio di sviluppare una polmonite, problemi che impongono di scegliere tra varie alternative, ognuna delle quali può comportare risultati clinici, sanitari ed economici specifici e differenti.

Il medico ha il vincolo morale e deontologico di offrire a ogni singolo paziente un procedimento diagnostico, una condotta terapeutica e un follow-up personalizzati, in grado di proteggere al meglio la vita e la qualità di vita del proprio cliente senza, tuttavia, perdere di vista l'ulteriore vincolo eti-

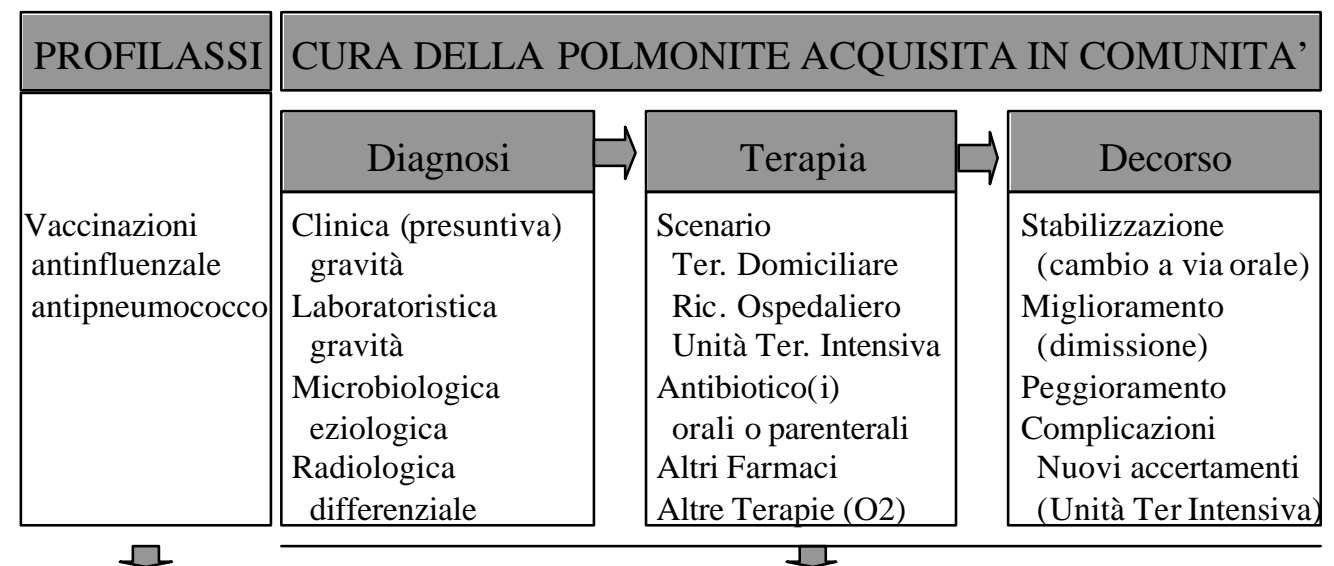

\begin{tabular}{|l|l|l|}
\hline \multicolumn{3}{|c|}{ Risultati e Conseguenze } \\
\hline Clinici (efficacia) & Soggettivi (utilità) & Economici (convenienza) \\
Guarigione & Qualità della Vita & Costi Diretti Sanitari \\
Morte & Gradimento della cura & Costi Diretti non Sanitari \\
Sequele, Invalidità & & Costi Indiretti \\
Resistenza all'antibiotico & & Costi sociali da resistenza \\
\hline
\end{tabular}

Figura 1

Sinossi delle possibili decisioni mediche nella gestione della CAP e delle relative conseguenze cliniche, sanitarie ed economiche. 
co e deontologico che consiste nel ricercare la massima efficienza ed equità allocative delle risorse sanitarie del paziente, della società e del Sistema Sanitario Nazionale (SSN).

L'analisi farmacoeconomica richiede vengano considerati i risultati clinici terminali e robusti della malattia. Nel caso della CAP riteniamo che il valore sanitario ed economico di una gestione del paziente debba essere valutato principalmente in base alle percentuali di guarigioni, di morti e di eventuali invalidità permanenti. Accanto a questi risultati sono considerati centrali anche le modificazioni della qualità di vita e il livello di gradimento della cura.

L'analisi dei costi dovrebbe essere esaustiva e non limitarsi ai costi diretti sanitari, o peggio ancora, ai soli costi del trattamento antibiotico, ma riguardare anche i costi diretti non sanitari e i costi indiretti che pesano sul paziente e sulla società.

L'uso degli antibiotici, soprattutto in patologie come le infezioni delle basse vie respiratorie, nel trattamento delle quali viene per lo più adottata una terapia empirica, comporta il rischio di insorgenza di resistenze batteriche: questo tipo di risultato supera $\mathrm{i}$ confini del singolo paziente, ma ha conseguenze sanitarie ed economiche rilevanti per la società e per i futuri pazienti.

La discussione seguente terrà conto dei nodi decisionali che possono influenzare queste tipologie di risultati clinici, oggettivi e soggettivi, sanitari ed economici.

In questo lavoro ci occupiamo essenzialmente della cura della polmonite acquisita in comunità, ma è opportuno sottolineare come un nodo decisionale importante sia quello della profilassi. I vaccini antiinfluenzali e antipneumococco sono risultati efficaci nel li- mitare i casi di CAP e nel ridurne la gravità soprattutto nelle persone ad alto rischio di complicazioni e nel personale sanitario (23). Sui problemi della profilassi non ci soffermiamo oltre, ripromettendoci di approfondire i risvolti farmaceoconomici di questo tema in un prossimo lavoro.

Le decisioni da prendere in relazione alla cura della CAP possono essere riferite a tre momenti differenti della gestione del paziente: la diagnosi, la terapia e il decorso dell'infezione.

\section{LA DIAGNOSI}

La CAP è definita come un'infezione acuta del parenchima polmonare, che si manifesta in pazienti non ospedalizzati o residenti in strutture assistenziali da non oltre 14 giorni prima dell'esordio dei sintomi, caratterizzata da alcuni sintomi tipici di un'infezione acuta e dalla presenza di infiltrato a rapida comparsa, rilevabile con una radiografia polmonare o con l'auscultazione. La diagnosi clinica si basa sulla presenza di un certo numero (almeno due, secondo alcuni autori) di sintomi di infezione acuta delle basse vie respiratorie, come la febbre o l'ipotermia, la sudorazione, la tosse con o senza produzione di sputo o cambiamento di colore delle secrezioni bronchiali, il dolore toracico e la comparsa di dispnea. La maggior parte dei pazienti presenta anche sintomi aspecifici come affaticamento, mialgie, dolori addominali, anoressia e cefalea $(23,25)$.

La comparsa acuta soprattutto dei segni specifici di infezione delle basse vie respiratorie dovrebbe far nascere il sospetto diagnostico che si tratti di polmonite, ma la diagnosi
Tabella 2

Discordanza tra medici nel rilevare e giudicare $i$ segni dell'esame obiettivo polmonare (Modificata da: Spiteri et al., 1988; citata da: Metlay JP et al., 1997) $(26,28)$

\begin{tabular}{|lcc|}
\hline Reperto polmonare & $\begin{array}{c}\text { Concordanza media tra } 2 \\
\text { medici - frequenza \% }\end{array}$ & $\begin{array}{c}\text { Probabilità di concordanza } \\
\text { casuale (k) }\end{array}$ \\
\hline \hline Tachipnea & 63 & 0,25 \\
Ridotta espansione del torace & 70 & 0,38 \\
Fremito tattile aumentato & 85 & 0,01 \\
Ottusità della percussione & 77 & 0,52 \\
Riduzione del soffio respiratorio & $---*$ & 0,43 \\
Sibili & 79 & 0,51 \\
Crepitii & 72 & 0,41 \\
Respiro bronchiale & $---*$ & 0,32 \\
Murmure vescicolare & $---*$ & 0,11 \\
\hline \hline
\end{tabular}

* Non calcolata quando 2 o più medici in un gruppo non ne hanno riportato la presenza o l'assenza 
definitiva spesso richiede l'esecuzione di una radiografia al torace.

Sintomi banali, come la tosse, sono l'occasione per una visita ambulatoriale dal medico. Da un'indagine condotta negli USA risulta che nel 1994 il sintomo tosse abbia comportato l'effettuazione di oltre 10 milioni di visite dei medici di primo livello, pari al $4 \%$ di tutte le visite mediche ambulatoriali (26). La polmonite risultò essere responsabile della tosse soltanto nel 5\% dei casi, collocandosi al quinto posto dopo la bronchite, le infezioni delle vie respiratorie superiori, l'asma e la sinusite.

La radiografia del torace è un esame standard per diagnosticare la polmonite acquisita in comunità e può fornire informazioni preziose anche sulla prognosi della malattia (27), oltre che a evidenziare condizioni coesistenti come un'ostruzione bronchiale o un versamento pleurico (19).

La radiografia del torace è un esame molto affidabile, sicuro, generalmente disponibile e relativamente poco costoso: perciò viene considerato come un esame standard nella valutazione di un paziente con sospetta polmonite. Dal punto di vista logistico, tuttavia, l'effettuazione della radiografia richiede che il paziente si rechi in ospedale o in un centro radiologico e ciò può rappresentare un problema oggettivo e/o soggettivo non trascurabile per alcuni pazienti.

Probabilmente alcuni medici continuano a far diagnosi di polmonite e a gestire pazienti affetti da polmonite senza l'ausilio di una radiografia del torace, mentre altri ricorrono di routine all' esame radiografico in ogni caso sospetto.

La situazione ideale sarebbe quella di un sistema validato di indicatori clinici capace di selezionare i pazienti per i quali l'esame radiografico è indispensabile da quelli per $\mathrm{i}$ quali è superfluo o inutile. Recentemente Metlay e collaboratori (26) hanno analizzato criticamente i lavori che avevano tentato di identificare una procedura diagnostica della polmonite basata sull'anamnesi e sull'esame oggettivo del paziente al fine di selezionare $i$ pazienti da sottoporre ad esame radiografico.

I medici spesso non concordano sulla presenza o assenza di un segno clinico indagato con l'esame obiettivo polmonare. La tabella 2 riporta, per ogni segno oggettivo indagato, le percentuali di concordanza di giudizio tra due medici come risultano da un'indagine effettuata da Spiteri e collaboratori su un gruppo di 24 medici (28). La precisione del dato clinico oggettivo non è in genere molto elevata e rende incerto il valore diagnostico e prognostico del singolo reperto o dell'insieme dei reperti. Analogo discorso si può fare per quanto riguarda la raccolta e l'utilizzo dei dati anamnestici.

Data la non elevata accuratezza della rilevazione e del giudizio medico, singoli sintomi o segni oggettivi non sono in grado di supportare o escludere una diagnosi di polmonite. Per tentare di superare queste difficoltà diversi autori hanno costruito algoritmi o regole predittive che utilizzano la presenza o assenza di vari dati anamnestici e dell'esame oggettivo (29-32).

Emerman e collaboratori (33) hanno analizzato comparativamente alcuni di questi algoritmi in relazione alla loro capacità di predire correttamente i risultati della radiografia. I risultati di questo studio, dove la prevalenza della polmonite era del 7\%, hanno evidenziato che i medici, utilizzando il solo giudizio clinico e senza far uso di regole specifiche, sbagliavano relativamente poco quando ritenevano che la radiografia non fosse necessaria, mentre l'errore aumentava quando ritenevano indispensabile l'esame radiografico per porre diagnosi di polmonite: questo risultato dimostra come vi sia un consumo indotto di esami radiografici non necessari. Ridurre tale "spreco" non è tuttavia facile.

L'uso di algoritmi validati, basati sulla presenza-assenza di vari sintomi o segni, consente di migliorare la stima della probabilità della diagnosi di polmonite senza ricorrere alla radiografia del torace: il più robusto di questi algoritmi richiede semplicemente l'assenza di ogni anomalia dei segni vitali per escludere la diagnosi di polmonite (32).

Non sono stati studiati algoritmi basati sull'uso combinato dell'anamnesi e dell'esame clinico oggettivo.

Lo studio di Metlay e collaboratori (26) conferma, tuttavia, che, nel caso sia necessario raggiungere un livello di certezza diagnostica nella gestione di un paziente con sospetta polmonite, occorre effettuare una radiografia del torace.

La diagnosi di gravità della polmonite è uno dei giudizi più importanti che deve dare il medico nel suo approccio iniziale al paziente. Infatti alla gravità della polmonite è correlato soprattutto il rischio di morte e dal livello di gravità spesso dipende, o almeno dovrebbe, la decisione di ricoverare o meno il paziente.

Lo sviluppo di modelli prognostici accurati e oggettivi può aiutare i medici a valutare il rischio dei loro pazienti affetti da polmonite e migliorare il processo decisionale che porta alla scelta del ricovero. Numerosi tentativi sono stati fatti in epoche recenti, ma $\mathrm{i}$ risultati sono stati deludenti per carenze di vario genere (34-40). 
Fine e collaboratori (11) hanno sviluppato, in questi ultimi anni, un sistema di valutazione prognostica del rischio di morte per pazienti affetti da CAP, caratterizzato dall'uso di pochi parametri facilmente disponibili anche a domicilio. Il metodo è stato sviluppato dapprima su un'ampia banca dati di oltre 14.000 casi e quindi validato utilizzando dati retrospettivi di oltre 38.000 pazienti ricoverati in vari ospedali statunitensi e con dati prospettici ottenuti su una coorte di circa 2.300 pazienti. $(6,11,34,41)$. Si tratta di un caso unico per dimensioni della casistica utilizzata e per rigore metodologico adottato.

L'algoritmo sviluppato da Fine e collaboratori classifica i pazienti affetti da CAP in cinque classi di rischio di morte, in base a un punteggio ottenuto con la semplice somma di punti pesati attribuiti all'età del paziente, ad alcuni segni clinici oggettivi, ad alcune patologie concomitanti, all'esame radiografico e a pochi parametri di laboratorio.

La figura 2 riporta lo schema dell'algoritmo di predizione, i parametri da rilevare, i punteggi relativi ottenuti con la validazione statistica del sistema. La valutazione si svolge in due fasi: la prima fase si può attuare con soli dati clinici ed è sufficiente per attribuire un soggetto alla Classe $\mathrm{I}^{\circ}$ (rischio di morte $<0,5 \%$ ) evitando per la maggior parte dei soggetti trattabili a domicilio l'effettuazione di esami di laboratorio e la radiografia del torace; la fase $\mathrm{II}^{\circ}$, invece, richiede l'effettuazione di pochi esami di laboratorio e dell'esame radiografico dei polmoni ma consente di discriminare accuratamente le Classi $\mathrm{II}^{\circ} \mathrm{-V}$ di rischio.

Le cinque classi di rischio sono caratte- rizzate da un range di mortalità contiguo, crescente dai livelli molto bassi $(<0,5 \%)$ della Classe $\mathrm{I}^{\circ}$ fino a mortalità superiori al $10 \%$ della Classe $\mathrm{V}^{\circ}$.

La tabella 3 riporta la distribuzione percentuali delle mortalità osservate nelle coorti di pazienti utilizzate dal gruppo di Fine per sviluppare e validare l'algoritmo predittivo. La tabella 4 indica, invece, la distribuzione percentuale dei soggetti attribuiti in base all'algoritmo a una delle cinque classi di rischio.

La possibilità di prevedere in modo robusto il rischio di morte di un paziente CAP consente di stabilire strategie decisionali che migliorino la qualità dell'assistenza e rendano complessivamente più efficiente il sistema. In particolare la previsione del rischio di mortalità nel caso della CAP viene utilizzata per decidere quali pazienti ospedalizzare e quali trattare a domicilio.

Non esistono indicazioni totalmente concordi e definitive su questo tema. Tuttavia la maggior parte degli autori ritiene che i soggetti che ricadono nelle Classi $\mathrm{I}^{\circ}$ e $\mathrm{II}^{\circ}$ di Fine non debbano essere ricoverati; la maggior parte dei soggetti in Classe III $^{\circ}$ potrebbero non essere ricoverati, mentre $\mathrm{i}$ soggetti in classe $\mathrm{IV}^{\circ} \mathrm{e} \mathrm{V}^{\circ}$ devono essere ricoverati. L'algoritmo di Fine non viene utilizzato per decidere quali pazienti trattare in Unità di Terapia Intensiva (UTI).

Lo strumento messo a punto da Fine appare molto robusto e si prospetta particolarmente utile per la gestione dei pazienti affetti da CAP. Alcuni gruppi di clinici italiani hanno iniziato a utilizzarlo soprattutto per selezionare i pazienti da ricoverare. Sarebbe,

\section{Figura 2 \\ Algoritmo di Fine per la stima predittiva del ri- schio di morte nei pazienti affetti da CAP. Suddivisione del livello di rischio in cinque classi, de- finite mediante si- stema ponderato di punteggivalidato applicabile in due fasi sequenziali (Modificatada: Fine MJ et al., 1997).(11)}

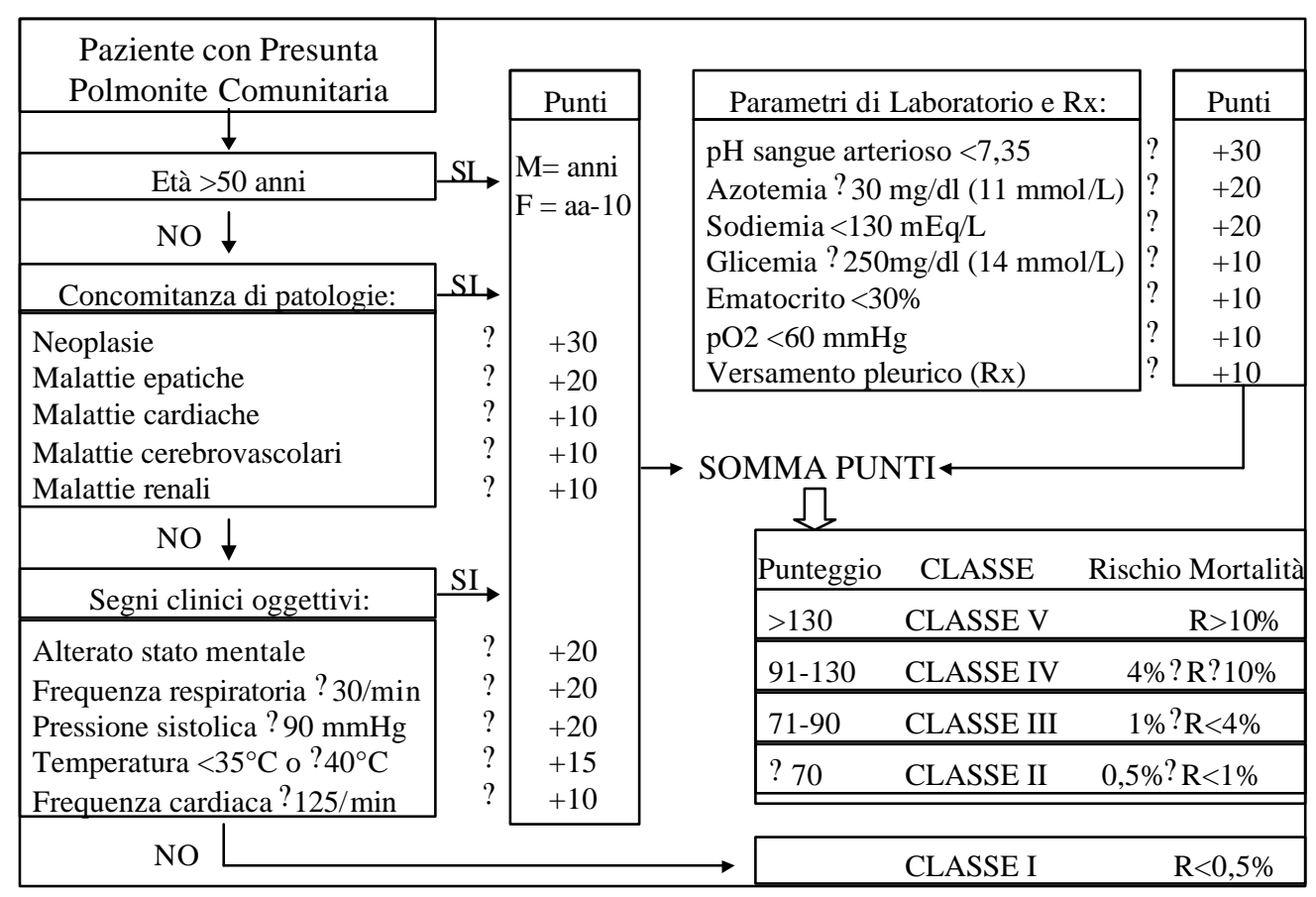




\begin{tabular}{|c|c|c|c|c|c|}
\hline \multirow[b]{2}{*}{ N. Pazienti } & \multicolumn{2}{|c|}{ Coorti MedisGroups } & \multicolumn{3}{|c|}{ Coorte Validazione PORT } \\
\hline & $\begin{array}{c}\text { Derivazione } \\
14.199\end{array}$ & $\begin{array}{c}\text { Validazione } \\
38.039\end{array}$ & $\begin{array}{c}\text { Ospedale } \\
1.343\end{array}$ & $\begin{array}{c}\text { Domicilio } \\
944\end{array}$ & $\begin{array}{l}\text { Totale } \\
2.287\end{array}$ \\
\hline Classe Rischio & \multicolumn{5}{|c|}{ Mortalità entro 30 giorni } \\
\hline (Punteggio) & $\%$ & $\%$ & $\%$ & $\%$ & $\%$ \\
\hline $\mathrm{I}^{\circ}$ & 0,4 & 0,1 & 0,5 & 0,0 & 0,1 \\
\hline $\mathrm{II}^{\circ}(<70)$ & 0,7 & 0,6 & 0,9 & 0,4 & 0,6 \\
\hline III $^{\circ}(71-90)$ & 2,8 & 2,8 & 1,2 & 0,0 & 0,9 \\
\hline $\mathrm{IV}^{\circ}(91-130)$ & 8,5 & 8,2 & 9,0 & 12,5 & 9,3 \\
\hline $\mathrm{V}^{\circ}(>130)$ & 31,1 & 29,2 & 27,1 & 0,0 & 27,0 \\
\hline Totale & 10,2 & 10,6 & 8,0 & 0,6 & 6,2 \\
\hline
\end{tabular}

Tabella 3

Correlazione tra le cinque classi di rischio definite secondo l'algoritmo predittivo di Fine e le distribuzioni percentuali della mortalità osservata entro 30 giorni nelle tre coorti di pazienti affetti da CAP utilizzate per lo sviluppo e la validazione del modello prognostico (Modificatada: Fine MJ et al., 1997) (11)

\begin{tabular}{|c|c|c|c|c|c|}
\hline \multirow[b]{2}{*}{ N. Pazienti } & \multicolumn{2}{|c|}{ Coorti MedisGroups } & \multicolumn{3}{|c|}{ Coorte Validazione PORT } \\
\hline & $\begin{array}{c}\text { Derivazione } \\
14.199\end{array}$ & $\begin{array}{c}\text { Validazione } \\
38.039\end{array}$ & $\begin{array}{c}\text { Ospedale } \\
1.343\end{array}$ & $\begin{array}{c}\text { Domicilio } \\
944\end{array}$ & $\begin{array}{l}\text { Totale } \\
2.287\end{array}$ \\
\hline Classe Rischio & \multicolumn{5}{|c|}{ Distibuzione percentuale intracoorte } \\
\hline (Punteggio) & $\%$ & $\%$ & $\%$ & $\%$ & $\%$ \\
\hline $\mathrm{I}^{\circ}$ & 9,7 & 8,0 & 13,8 & 62,3 & 33,7 \\
\hline $\mathrm{II}^{\circ}(<70)$ & 17,0 & 15,2 & 17,3 & 25,9 & 20,9 \\
\hline $\mathrm{III}^{\circ}(71-90)$ & 18,5 & 17,8 & 18,9 & 7,6 & 14,3 \\
\hline $\mathrm{IV}^{\circ}(91-130)$ & 33,1 & 34,5 & 33,2 & 4,2 & 21,2 \\
\hline$V^{\circ}(>130)$ & 21,7 & 24,5 & 16,8 & 0,0 & 9,9 \\
\hline Totale & 100,0 & 100,0 & 100,0 & 100,0 & 100,0 \\
\hline
\end{tabular}

Tabella 4

Distribuzioni percentuali entro le cinque classi di rischio definite secondo l'algoritmo predittivo di Fine dei pazienti inclusi nelle tre coorti utilizzate per lo sviluppo e la validazione del modello prognostico (Modificata e rielaborata da: Fine MJ et al., 1997)(11)

tuttavia, opportuno che lo strumento venisse sottoposto a validazione in relazione alla popolazione italiana.

La classificazione di Fine non utilizza né la conta dei globuli bianchi, né esami microbiologici. D'altra parte l'obiettivo di questo strumento è quello di fornire una prognosi di mortalità affidabile e semplice da utilizzare.

Il clinico nel suo iter diagnostico, soprattutto differenziale, su pazienti che manifestano segni di infezione delle basse vie respiratorie, spesso utilizza esami di laboratorio per definire lo stato infiammatorio e la gravità del paziente ed esami microbiologici per tentare di stabilire l'eziologia dell'infezione polmonare e stimare la sensibilità agli antibiotici dei patogeni eventualmente isolati. Per effettuare questi esami il paziente deve essere ricoverato, oppure deve recarsi in un laboratorio, a meno che non sia inserito in un programma di assistenza domiciliare che provvede all'effettuazione di questi esami senza dover spostare il paziente. I pazienti ricoverati sono sottoposti, anche per la loro gravità clinica, a una maggior quantità di esami e ad approfonsimenti diagnostici maggiori.

Le diverse linee-guida pubblicate prendono in considerazione questi punti e non sempre concordano totalmente: per un' approfondita discussione si rimanda ai lavori originali (19, 20) Le linee-guida della British Thoracic Society prevedono che tutti i pazienti ricoverati per sospetta CAP vengano sottoposti a una radiografia del torace, all'esame emocromocitometrico con conta completa e formula leucocitaria, all'esame delle urine, alla determinazione dell'azotemia e degli elettroliti, ai tests di funzionalità epatica, alla emocoltura e alla coltura dello sputo e del liquido pleurico se presente. L'emogasanalisi dovrebbe essere eseguita in tutti i pazienti che presentano una polmonite, tranne che nei casi di modesta gravità. Nei casi gravi, inoltre, si dovrebbe procedere con maggior impegno alla ricerca 
dell'agente eziologico mediante uno striscio dello sputo e colorazione di Gram e mediante indagini sierologiche, la ricerca di antigeni dello peumococco nello sputo, nel sangue e nell'urine. Indagini invasive possono essere prese in considerazione nei pazienti che per la gravità delle loro condizioni cliniche vengono trasferiti in UTI (20).

Altre linee-guida sottolineano l'importanza di eseguire, anche nei pazienti non ricoverati, almeno un esame microscopico con colorazione di Gram per individuare i microrganismi presenti nello sputo (23).

Le opinioni degli esperti sulla necessità di effettuare indagini microbiologiche e sulla loro costo/efficacia sono discordanti: la maggior parte dei lavori scientifici pubblicati evidenziano limitati vantaggi e non risulta che sia mai stata provata chiaramente una convenienza in termini di rapporto costo/efficacia. Tuttavia, generalmente è riconosciuta l'importanza di espletare ogni tentativo ragionevole per giungere a stabilire una diagnosi eziologica della polmonite nei pazienti ricoverati, soprattutto con l'obiettivo di migliorare l'utilizzo degli antibiotici disponibili e di scegliere la strategia terapeutica maggiormente costo/efficace.

Secondo le recenti linee-guida ESOCAP, la maggior parte dei pazienti adulti affetti da infezione delle basse vie respiratorie acquisite in comunità possono essere gestiti senza ricorrere ad alcuna indagine strumentale o di laboratorio. Vengono, peraltro, fissate le condizioni che inducono a raccomandare o a non raccomandare l'effettuazione di una radiografia del torace, di un esame microscopico sullo sputo, di altri esami ematochimici e microbiologici, nonché di funzionalità polmonare (24). Tali raccomandazioni sono riassunte nella tabella 5 .

\section{L'IMPOSTAZIONE DELLA TERAPIA}

La strategia terapeutica complessiva della CAP si articola su alcuni nodi decisionali fondamentali: trattare a domicilio o ricoverare il paziente e, in questo caso, ricoverare in corsia normale o in Unità di Terapia Intensiva; scegliere l'antibiotico o l'associazione di antibiotici; stabilire la posologia e le modalità di somministrazione orale o parenterale (intramuscolare, endovenosa a bolo o per infusione); considerare l'eventuale associazione di altri farmaci o di altre terapie (ossigenoterapia).

Ognuna di queste scelte comporta differenti ripercussioni sul bilancio finale delle risorse consumate. Evitare un ricovero comporta tendenzialmente un notevole risparmio per il SSN e per la società e anche significativi miglioramenti della qualità di vita del paziente e dei familiari. Come ripetutatmente osservato, la decisione di ricoverare una sospetta polmonite di origine comunitaria dovrebbe essere guidata essenzialmente dalla gravità della malattia e in particolare dal rischio di morte. A questo scopo sempre più frequentemente viene proposto di utilizzare l'algoritmo di Fine (11) per decidere razionalmente quali pazienti debbano essere ricoverati e quali possano essere trattati a domicilio. È noto, tuttavia, che la decisione di ricoverare un paziente con sospetta CAP viene spesso condizionata da fattori socio-culturali e ambientali, come l'ansia evocata dal-
Tabella 5

Raccomandazioni delle linee-guida ESOCAP sulle indagini radiografiche, microbiologiche, laboratoristiche e funzionali da effettuare, da non effettuare o da prendere in considerazione nei pazienti affetti da CAP in funzione di alcune condizioni o fattori di rischio (Modificata da: ESOCAP, 1998) (24)

\begin{tabular}{|c|c|c|c|c|}
\hline & Rx torace & \begin{tabular}{|c||} 
Esame \\
microbiologico \\
dello sputo
\end{tabular} & \begin{tabular}{|c|} 
Conta GB, \\
Proteina-CR, \\
emocolture, \\
sierologia, \\
antigeni \\
pneumococco \\
e legionella \\
\end{tabular} & $\begin{array}{l}\text { Test } \\
\text { funzionalità } \\
\text { polmonare }\end{array}$ \\
\hline $\begin{array}{l}\text { Nessun fattore di rischio per } \\
\text { gravità infezione o patogeni } \\
\text { inusuali }\end{array}$ & NR & NR & NR & NR \\
\hline $\begin{array}{l}\text { Fattori di rischio per gravità } \\
\text { infezione }\end{array}$ & $\mathrm{PiC}$ & NR & $\mathrm{PiC}$ & NR \\
\hline $\begin{array}{l}\text { Fattori di rischio per } \\
\text { patogeni inusuali }\end{array}$ & NR & $\mathrm{R}$ & NR & NR \\
\hline $\begin{array}{l}\text { Insuccesso prima terapia } \\
\text { empirica }\end{array}$ & $\mathrm{R}$ & $\mathrm{R}$ & $\mathrm{PiC}$ & $\mathrm{PiC}$ \\
\hline Segni di focolaio polmonare & $\mathrm{R}$ & NR & NR & NR \\
\hline Respiro asmatico, atopia & NR & NR & NR & $\mathrm{R}$ \\
\hline
\end{tabular}


l'idea della malattia, la disponibilità di ospedali nell' area geografica, la disponibilità del supporto collaborativo della famiglia o di gruppi di volontariato, la disponibilità di programmi di assistenza domiciliare integrata (ADI).

Talvolta nel decidere il ricovero gioca un ruolo critico la necessità di attuare una terapia antibiotica aggressiva per via endovenosa. Quando l'unico motivo per decidere il ricovero fosse quello della somministrazione parenterale, si dovrebbe prendere in seria considerazione l'opzione alternativa di trattare il paziente a domicilio utilizzando il supporto di programmi di assistenza domiciliare in grado di garantire la terapia antibiotica iniettiva anche per via endovenosa: questa strategia è sicuramente meno costosa del ricovero ospedaliero (42).

La scelta dell' antibiotico o dell' associazione di antibiotici da utilizzare inizialmente in un paziente affetto da CAP è uno dei nodi decisionali fondamentali. L'ampio ventaglio di antibiotici disponibili, l'evoluzione epidemiologica dei patogeni e delle resistenze e l'interferenza di innumerevoli interessi economici rende la scelta non facile.

La terapia antibiotica iniziale delle CAP è nella stragrande maggioranza dei casi di tipo empirico-ragionato e solo eccezionalmente può essere mirata. Infatti, l'isolamento dell'agente eziologico è difficoltoso e risulta possibile soltanto in una percentuale limitata di pazienti (30-40\%); inoltre richiede comunque tempi lunghi, quasi sempre non compatibili con l'esigenza clinica di iniziare tempestivamente la terapia antibiotica.

La scelta empirica dell'antibiotico si dovrebbe comunque basare sulle conoscenze dell'epidemiologia microbiologica locale delle polmoniti e sull'andamento locale delle resistenze batteriche ai vari antibiotici. Purtroppo gli osservatori epidemiologici sui microrganismi patogeni non sono molti e spesso non sono diffusi capillarmente sul territorio. Inoltre i dati prodotti non sempre sono omogenei e confrontabili e spesso non sono facilmente accessibili. In letteratura si trovano pubblicate numerose ricerche di epidemiologia microbiologica, ma spesso considerano un solo patogeno.

Come riferimento per la scelta empirica di un antibiotico vengono generalmente indicate alcune rassegne di epidemiologia microbiologica che forniscono dati talvolta vecchi, quindi non più aderenti alla realtà attuale, talvolta estremamente variabili. Nella tabella 6 riportiamo come esempio due delle rassegne più citate anche dalle linee-guida (43-44). Dati di questo tipo forniscono solo un quadro macroscopico del fenomeno e consentono di fare una prima cernita sulle classi di antibiotico da utilizzare nelle CAP, senza tuttavia aiutare nella scelta più specifica di quale antibiotico utilizzare come prima linea e soprattutto quale molecola scegliere in una classe di antibiotici.

Le diverse linee-guida cercano di risolvere il problema della scelta dell'antibiotico attraverso operazioni di "consensus" tra esperti. Tuttavia, nella produzione di consensus su opinioni controverse e ricche di implicazioni economiche come queste, non sempre i criteri guida sono completamente espliciti e trasparenti. Le opinioni e le esperienze personali degli esperti, le condizioni e i vincoli del sistema assistenziale sanitario, in cui si prospetta l'applicazione concreta delle linee-guida,

\begin{tabular}{|lccc|}
\hline & $\begin{array}{c}\text { MacFarlane (43) } \\
\text { Microrganismi }\end{array}$ & \multicolumn{2}{c|}{ Woodhead (44) } \\
\cline { 3 - 4 } & (Casistiche varie) & $\begin{array}{c}<65 \text { anni } \\
\%\end{array}$ & $>65$ anni \\
$\%$
\end{tabular}

Tabella 6

Distribuzioni percentuali dei principali patogeni responsabili della CAP secondo due diverse rassegne sulla epidemiologia microbiologica pubblicate negli anni '80 e '90. (43, 44) 
Tabella 7

Scelta dell'antibiotico nel trattamento domiciliare del paziente con polmonite acquisita in comunità secondo le lineeguida ISDA e $\operatorname{ESOCAP}(23,24)$

\begin{tabular}{|c|c|c|}
\hline & Linee guida ISDA & Linee guida ESOCAP \\
\hline $\begin{array}{l}\text { Antibiotici di prima } \\
\text { scelta }\end{array}$ & $\begin{array}{l}\text { Macrolidi } \\
\text { Fluorochinoloni } \\
\text { Doxiciclina }\end{array}$ & Aminopenicillina \\
\hline $\begin{array}{l}\text { Alternative alla prima } \\
\text { scelta }\end{array}$ & $\begin{array}{l}\text { Amoxicillina/clavulanato } \\
\text { Alcune cefalosporine orali } \\
\text { (cefuroxime, cefpodoxime, } \\
\text { cefprozil) }\end{array}$ & \begin{tabular}{|l} 
Tetracicline \\
Cefalosporine orali \\
Fluorochinoloni \\
Macrolidi \\
Streptogramine orali
\end{tabular} \\
\hline \multicolumn{3}{|l|}{ Condizioni particolari } \\
\hline $\begin{array}{l}\text { Polmonite non grave in } \\
\text { soggetti giovani adulti, } \\
\text { specialmente durante } \\
\text { epidemie di } \\
\text { Mycoplasma } \\
\end{array}$ & Doxiciclina & Macrolidi \\
\hline $\begin{array}{l}\text { Elevata frequenza di } \\
\text { ceppi betalattamasi- } \\
\text { produttori (Haemophilus } \\
\text { influenzae), BPCO o } \\
\text { insuccesso con } \\
\text { aminopenicillina }\end{array}$ & $\begin{array}{l}\text { Fluorochinoloni } \\
\text { Amoxicillina/clavulanato }\end{array}$ & $\begin{array}{l}\text { Aminopenicilline }+ \text { Blattamasi- } \\
\text { inibitore }\end{array}$ \\
\hline
\end{tabular}

Tabella 8

Scelta degli antibiotici per il trattamento ospedaliero di pazienti affetti da polmonite acquisita in comunità secondo le linee-guida ISDA $e$ ESOCAP. $(23,24)$

\begin{tabular}{|c|c|c|}
\hline & Linee guida ISDA & Linee guida ESOCAP \\
\hline $\begin{array}{l}\text { Pazienti in degenza } \\
\text { ordinaria }\end{array}$ & $\begin{array}{l}\text { Una betalattamina } \\
\pm \\
\text { un macrolide } \\
\text { o un fluorochinolone da solo In } \\
\text { alternativa: } \\
\text { Cefuroxime } \\
\pm \\
\text { un macrolide o } \\
\text { Azitromicina da sola }\end{array}$ & $\begin{array}{l}\text { Una cefalosporina } \mathrm{II}^{\circ} \text { gen iv } \\
\text { o una cefalosporine } \text { III }^{\circ} \text { gen iv } \\
\text { o una } \beta \text {-lattamina/inibitore- } \beta- \\
\text { latt. iv od orale } \\
\text { o Benzilpenicillina iv } \\
\text { o Amoxicillina iv } \\
\text { o Ampicillina iv } \\
\pm \\
\text { un macrolide iv od orale }\end{array}$ \\
\hline \multicolumn{3}{|l|}{ Condizioni particolari } \\
\hline $\begin{array}{l}\text { Bronchiectasie o altre } \\
\text { malattie con alterazione } \\
\text { strutturale del polmone }\end{array}$ & $\begin{array}{l}\text { Penicilline antipseudomonali o } \\
\text { carbapenemici o cefepime } \\
+ \\
\text { macrolide o fluorochinolone } \\
+ \\
\text { aminoglicoside }\end{array}$ & $\begin{array}{l}\text { Amoxicillina/clavulanato iv ad } \\
\text { alte dosi }\end{array}$ \\
\hline Allergia alla penicillina & $\begin{array}{l}\text { Fluorochinolone } \\
\pm \\
\text { clindamicina }\end{array}$ & Clindamicina iv \\
\hline Sospetto di aspirazione & \begin{tabular}{|l} 
Fluorochinolone \\
+ \\
Clindamicina \\
o una B-lattamina/inibitore-ß3-latt.
\end{tabular} & $\begin{array}{l}\text { Amoxicillina/clavulanato iv ad } \\
\text { alte dosi }\end{array}$ \\
\hline $\begin{array}{l}\text { Paziente in Terapia } \\
\text { Intensiva }\end{array}$ & $\begin{array}{l}\text { Eritromicina o azitromicina o un } \\
\text { fluorochinolone } \\
+ \\
\text { cefotaxime o ceftriaxone o una } \\
\text { b-lattamina/inibitore-B3-lattamasi }\end{array}$ & $\begin{array}{l}\text { Cefalosporine } \mathrm{II}^{\circ} \text { o } \mathrm{III}^{\circ} \\
\text { generazione iv alte dosi } \\
+ \\
\text { Fluorochinoloni o macrolidi iv } \\
\pm \\
\text { Rifampicina iv }\end{array}$ \\
\hline
\end{tabular}


sono fattori che pesano nel determinare le indicazioni degli antibiotici da utilizzare come prima linea o come opzioni alternative o nei casi particolari. Nelle linee-guida pubblicate tale problema traspare nettamente. A tal proposito si confrontino le indicazioni sulla scelta degli antibiotici nella CAP delle due più recenti linee-guida, quella dell'ISDA (23) prodotta negli Stati Uniti e quella dell'ESOCAP (24) prodotta in Europa (vedi tabelle 7 e 8). Le differenze di indicazione sono significative sia per quanto riguarda il trattamento antibiotico dei pazienti CAP a domicilio sia per quanto riguarda $\mathrm{i}$ ricoverati.

L'introduzione di linee-guida dovrebbe essere accompagnata da una validazione sul campo per accertare quale impatto sulla salute e sull'efficienza dell' assistenza comporti la loro adozione su ampia scala. Per quanto riguarda le linee-guida sulla CAP, scarsi sono i dati di verifica finora prodotti, i quali, tuttavia indicano come le conseguenze economiche siano complesse e non facilmente prevedibili (45).

Lo stile prescrittivo è solo in parte influenzato dalla pubblicazione di linee-guida. D'altra parte esiste un'elevata variabilità nello stile prescrittivo degli antibiotici in generale e nel caso delle CAP in particolare. La differenza di stile prescrittivo è sicuramente elevata tra medici, in funzione della loro collocazione assistenziale e della loro qualificazione professionale, ma è anche notevole nelle diverse aree geografiche e nelle diverse nazioni, come dimostra un'indagine recente effettuata in cinque paesi europei (16).

È noto che i medici italiani hanno un comportamento prescrittivo degli antibiotici significativamente diverso da quello di altri Paesi europei, anche confinanti. In generale, in Italia si fa uso frequente di antibiotici per via intramuscolare e poco per via orale, soprattutto nel caso di infezioni ritenute gravi come le polmoniti: tale abitudine sembra prescindere da una valutazione oggettiva e ponderata della gravità del caso e dipende piuttosto dai livelli d'ansia che la malattia induce nel paziente e nel medico. Inoltre, in Italia è molto diffuso il convincimento che le malattie infettive che possono avere conseguenze gravi debbano essere trattate con antibiotici iniettivi, indipendentemente dalla concreta valutazione del rischio nel singolo paziente.

Nel trattamento antibiotico delle CAP, sia a domicilio sia in ospedale, il medico italiano sceglie prevalentemente di utilizzare una cefalosporina di terza generazione. Questo comportamento prescrittivo è nettamente in contrasto con le indicazioni delle linee-guida. Questa constatazione è generalmente occasione per critiche alla classe medica italiana per- ché il suo comportamento sarebbe causa di costi farmaceutici non giustificabili e del rischio di insorgenza di resistenze.

Su questo punto, tuttavia, sarebbe urgente avviare indagini per verificare le conseguenze sanitarie ed economiche di tale diffuso stile prescrittivo. La semplice constatazione che il comportamento prescrittivo dei medici italiani è nettamente diverso da quello dei colleghi inglesi non può, ovviamente, essere assunta come dimostrazione della tesi secondo cui l'uso italiano di antibiotici sarebbe meno costo/efficace di quello inglese. I pochi dati comparativi disponibili, come quelli prodotti dal gruppo collaborativo ESOCAP, dimostrerebbero semmai che la situazione italiana è nettamente migliore di quella inglese per aspetti fondamentali come la mortalità per infezioni polmonari e la percentuale di ricoveri tra i pazienti affetti da CAP (16). Inoltre, in Italia la prevalenza attuale di ceppi di penumococco resistenti alla penicillina è nettamente inferiore a quella di altri paesi che prediligono gli antibiotici orali a quelli iniettivi. In definitiva, quindi, ogni giudizio sul comportamento prescrittivo di una classe medica potrebbe avere maggior peso se fosse supportato da una qualche validazione farmacoeconomica che valuti tutte le conseguenze sanitarie ed economiche rilevanti.

\section{IL DECORSO}

Stabilita la diagnosi e la terapia iniziale, il paziente affetto da CAP deve essere monitorato attentamente, soprattutto durante i primi giorni di terapia per evidenziare l'evoluzione positiva o negativa della situazione clinica e adottare le misure più idonee.

I casi a evoluzione positiva tendono alla stabilizzazione clinica dopo pochi giorni di terapia per poi volgere verso un miglioramento progressivo, talvolta con qualche oscillazione, e approdare alla guarigione. Nel caso in cui il trattamento iniziale sia stato aggressivo e abbia comportato l'utilizzo di uno o più antibiotici per via parenterale, appena raggiunta la stabilità clinica si deve considerare l'opzione di semplificare il trattamento antibiotico riducendo il numero di farmaci e passando alla via orale (46-49). Questa strategia, introdotta alcuni anni or sono e conosciuta come "switch therapy" o "terapia sequenziale", comporta alcuni vantaggi rilevanti anche sotto il profilo farmacoeconomico: semplifica il trattamento, facilita la dimissione e il trattamento domiciliare, migliora la qualità di vita del paziente, riduce i costi della terapia antibiotica perché risparmia sia sui costi d'acquisto dei farmaci sia sui costi di somministrazione. 
I casi che non si stabilizzano in 2-3 giorni richiedono una revisione diagnostica ed eventualmente terapeutica. Occorre, infatti, stabilire se la diagnosi iniziale è corretta oppure sbagliata, se sono insorte complicazioni, se l'antibiotico scelto e la posologia adottata possono essere considerate adeguate nonostante il ritardo di comparsa della risposta, oppure se la scelta è stata inadeguata perché l'infezione è sostenuta da un patogeno non sensibile o divenuto resistente all'antibiotico (23).

Alcuni casi si presentano immediatamente come gravi e altri evolvono più o meno rapidamente verso condizioni cliniche critiche: in questi casi si pone il problema di decidere l'eventuale invio in Unità di Terapia Intensiva.

Il concetto di stabilizzazione clinica del paziente affetto da polmonite non è univocamente definito. Tuttavia, la maggior parte dei libri di testo e delle linee-guida fondano il concetto di stabilità clinica del paziente riferendosi alla normalizzazione della frequenza cardiaca, della pressione arteriosa sistolica, della frequenza respiratoria, della temperatura, dell'ossigenzazione, della capacità di alimentarsi e dello stato mentale (25, 50, 51).

Recentemente Halm e collaboratori (50) hanno studiato, in una coorte di pazienti adulti affetti da CAP, il tempo necessario per la normalizzazione di questi parametri. Nella tabella 9 vengono riportate le percentuali di soggetti che presentano alterazioni dei parametri vitali al primo giorno di trattamento e la distribuzione, espressa come mediana e come range interquartile, della durata (giorni) dell'instabilità. Come si può facilmente rilevare, la maggioranza dei soggetti recupera stabilità dei segni vitali entro 2-4 giorni dall'inizio del trattamento.

La durata dell'instabilità clinica dipende dai criteri stabiliti per definire la stabilizzazione. Non c'è accordo universale sui livelli soglia di temperatura, frequenza cardiaca e saturazione di ossigeno da assumere come riferimento per la normalizzazione. Nella tabella 10 sono riportate cinque differenti combinazioni (A-E) dei tre criteri fondamentali (temperatura, saturazione di ossigeno e frequenza del respiro) come stabiliti da vari autori: ovviamente, assumendo criteri maggiormente restrittivi, la durata dell'instabilità aumenta. Inoltre, la durata del periodo di instabilità clinica è in qualche misura direttamente correlata anche con la gravità iniziale della malattia, ovvero con il livello di rischio di morte secondo la classificazione di Fine. Si noti come le prime tre classi di Fine, che rappresentano i pazienti con polmoniti di grado lieve-moderato per le quali non è generalmente richiesto il ricovero, siano accomunate in questa relazione: le differenze nella durata dell'instabilità emer-

\begin{tabular}{|c|c|c|c|c|}
\hline & Criterio di stabilità clinica & ${\text { Instabili } \mathrm{I}^{\circ}}$ & Durata de & ilità (giorni) \\
\hline & & $\%$ & Mediana & $\begin{array}{c}\text { Range } \\
\text { Interquartile }\end{array}$ \\
\hline & Pressione sistolica $>90 \mathrm{mmHg}$ & 7 & 2 & $2-3$ \\
\hline & Frequenza cardiaca $<100 \mathrm{~b} / \mathrm{min}$ & 56 & 2 & $2-3$ \\
\hline & Frequenza respiratoria & & & \\
\hline & $<24 \mathrm{resp} / \mathrm{min}$ & 49 & 3 & $2-4$ \\
\hline & $<22 \mathrm{resp} / \mathrm{min}$ & 71 & 3 & $2-6$ \\
\hline & $<20 \mathrm{resp} / \mathrm{min}$ & 78 & 4 & $3-7$ \\
\hline & Temperatura corporea & & & \\
\hline & $<38,3{ }^{\circ} \mathrm{C}$ & 46 & 2 & $2-3$ \\
\hline & $<37,8{ }^{\circ} \mathrm{C}$ & 63 & 3 & $2-4$ \\
\hline & $<37,2{ }^{\circ} \mathrm{C}$ & 80 & 3 & $2-6$ \\
\hline Tabella 9 & Saturazione ossigeno & & & \\
\hline Distribuzione della & $<90 \%$ & 23 & 3 & $2-6$ \\
\hline $\begin{array}{l}\text { durata (giorni) di } \\
\text { instabilitòclinica }\end{array}$ & $<92 \%$ & 31 & 3 & $2-6$ \\
\hline dei pazienti affetti & $<94 \%$ & 39 & 4 & $2-8$ \\
\hline da CAP (modificata & Capacità di alimentarsi & 11 & 2 & $2-8$ \\
\hline $\begin{array}{l}\text { aa: Halm EA et al., } \\
\text { 1998)(50) }\end{array}$ & Stato mentale normale & 8 & 3 & $2-4$ \\
\hline
\end{tabular}




\begin{tabular}{|c|c|c|c|c|c|}
\hline & \multicolumn{5}{|c|}{ Criteri stabilità } \\
\hline & A & B & $\mathrm{C}$ & $\mathrm{D}$ & $\mathrm{E}$ \\
\hline Temperatura $\left({ }^{\circ} \mathrm{C}\right)$ & $<38,3$ & $<37,8$ & $<37,2$ & $<37,2$ & $<37,2$ \\
\hline Saturazione $\mathrm{O} 2(\%)$ & $<90$ & $<90$ & $<92$ & $<92$ & $<94$ \\
\hline \multirow[t]{2}{*}{ Freq Respiro (Resp/min) } & $<24$ & $<24$ & $<24$ & $<20$ & $<20$ \\
\hline & \multicolumn{5}{|c|}{ Giorni di instabilità clinica: Mediane (range interquartile) } \\
\hline Campione totale & $3(2-5)$ & $3(2-6)$ & $5(3-8)$ & $6(4-13)$ & $7(4-17)$ \\
\hline \multicolumn{6}{|l|}{ Rischio mortalità } \\
\hline $\mathrm{I}^{\circ}-\mathrm{III}^{\circ}$ classe & $3(2-4)$ & $3(2-5)$ & $4(3-8)$ & $6(3-12)$ & $6(4-17)$ \\
\hline $\mathrm{IV}^{\circ}$ classe & $3(2-7)$ & $4(2-7)$ & $6(3-9)$ & $7(3-16)$ & $9(4-17)$ \\
\hline $\mathrm{V}^{\circ}$ classe & $5(3-9)$ & $6(3-9)$ & $7(4-11)$ & $10(6-11)$ & $13(7-17)$ \\
\hline
\end{tabular}

Tabella 10

Uso di differenti criteri per definire la stabilità clinica e suoi effetti sulla distribuzione della durata (giorni) di instabilità clinica in funzione delle cinque classi di rischio di morte definite secondo l'algoritmo predittivo di Fine (Modificata da: Halm EA et al., 1998) (50)

gono con il passaggio alle classi $\mathrm{IV}^{\circ} \mathrm{e}^{\circ}$ di Fine.

Il $14-15 \%$ circa dei pazienti in condizioni di instabilità clinica si aggrava fino a dover essere inviato in UTI. I dati riportati in tabella 11 indicano che tale percentuale non è praticamente correlata con le differenti definizioni di stabilità clinica. Al contrario, la percentuale di pazienti $(0,3-1 \%)$ che viene inviato in UTI dopo aver raggiunto la stabilità clinica è inversamente correlata con le differenti definizioni di stabilità: più i criteri sono restrittivi, minore è la probabilità che il soggetto abbia un'inversione di tendenza e che peggiori fino a dover essere inviato in UTI. Passando dalla definizione $\mathrm{A}$, meno restrittiva, alla definizione $\mathrm{E}$, più restrittiva, la percentuale di pazienti che prolunga per più di un giorno la degenza in ospedale dopo aver raggiunto la stabilità clinica e il numero di gior- ni di degenza dopo aver raggiunto la stabilità diminuiscono, mentre aumenta la percentuale di pazienti che viene inserito in un programma di "switch therapy" entro un giorno dall'essersi stabilizzato (tabella 11).

Tutte queste correlazioni indicano, nella sostanza, che le cinque definizioni di stabilità rappresentano realmente una differente graduazione clinica, percepita dal medico come parametro importante per prendere decisioni fondamentali nella gestione del paziente affetto da CAP, come quella di adottare la "switch therapy" o quella di dimettere il paziente determinando, quindi, la durata del ricovero ospedaliero (50). Queste decisioni, com'è intuitivo, dipendono dalla costatazione che le condizioni cliniche del paziente non solo sono stabilizzate ma sono anche in via di definitiva risoluzione. Il livello oggettivo di normalizzazione clinica non è, tuttavia, suf-

\begin{tabular}{|c|c|c|c|c|c|}
\hline & \multicolumn{5}{|c|}{ Criteri di stabilità } \\
\hline & A & B & $\mathrm{C}$ & $\mathrm{D}$ & $\mathrm{E}$ \\
\hline Temperatura $\left({ }^{\circ} \mathrm{C}\right)$ & $<38,3$ & $<37,8$ & $<37,2$ & $<37,2$ & $<37,2$ \\
\hline Saturazione $\mathrm{O} 2(\%)$ & $<90$ & $<90$ & $<92$ & $<92$ & $<94$ \\
\hline Freq Respiro (Res/min) & $<24$ & $<24$ & $<24$ & $<20$ & $<20$ \\
\hline \multicolumn{6}{|l|}{ Inviati in UTI (\%) } \\
\hline Durante linstabilità & 14 & 14 & 14 & 15 & 15 \\
\hline Dopo raggiunta stabilità & 1 & 1 & 0,6 & 0,5 & 0,3 \\
\hline \multicolumn{6}{|c|}{ Prolungamento degenza > un giorno dopo raggiunta stabilità clinica } \\
\hline$\%$ pazienti & 86 & 83 & 77 & 66 & 65 \\
\hline \multicolumn{6}{|c|}{ Giorni degenza dopo raggiunta stabilità } \\
\hline Mediana (interquartile) & $4(2-7)$ & $4(2-7)$ & $3(2-6)$ & $3(1-6)$ & $3(1-6)$ \\
\hline \multicolumn{6}{|c|}{ Pazienti in Switch Therapy entro un giorno dopo stabilità } \\
\hline$\%$ pazienti & 29 & 35 & 40 & 46 & 44 \\
\hline
\end{tabular}

Tabella 11

Uso di differenti criteri per definire la stabilità clinica e suoi effetti percentuale di pazienti inviati in UTI, sul prolungamento della degenza, nonché sulla percentuali di pazienti in "Switch therapy" entro un giorno dalla raggiunta stabilità (Modificata da: Halm EA et al., 1998)(50) 
ficiente a determinare tali decisioni nel medico: egli deve convincersi, utilizzando la sua percezione soggettiva di questa evoluzione, che la terapia antibiotica può essere semplificata $\mathrm{e}$ che il paziente può essere dimesso senza che corra rischi inaccettabili.

La letteratura internazionale e alcune rassegne critiche evidenziano che la durata del ricovero di un paziente affetto da CAP varia molto non solo da nazione a nazione, da regione a regione, ma anche da ospedale a ospedale di una stessa regione geografica e addirittura da reparto a reparto all'interno di uno stesso ospedale (52-55). Queste differenze persistono anche dopo aver eliminato le influenze della gravità della malattia, della comorbidità e delle caratteristiche dell'ospedale. Pertanto le differenze sono presumibilmente dovute all'incertezza clinica e/o alle differenze di stile nella conduzione clinica dei vari medici $(53,54,56,57)$. Inoltre alcune volte il medico prolunga la degenza del paziente con polmonite oltre il momento della raggiunta stabilità clinica spesso per motivi non strettamente correlati all'evoluzione dell'infezione.

Un ricovero breve è senza dubbio conveniente per l'ospedale e in definitiva per il SSN e la società, qualora tutto il sistema dell'assistenza sanitaria abbia raggiunto la masssima efficienza. D'altra parte il medico si pone il problema di capire se la dimissione precoce pregiudichi la futura evoluzione clinica del paziente affetto da polmonite e lo ponga in una condizione di rischio inaccettabile $(52$, $54,55,57-62)$. Una recente indagine ha evidenziato che ridurre i tempi di degenza e dimettere il paziente non appena stabilizzato non comporta alcun peggioramento degli esiti sanitari nei pazienti ricoverati per CAP (57). Viene, quindi, confermata l'opportunità di ridurre la durata media della degenza per CAP, utilizzando la semplice strategia di dimettere il paziente entro pochissimi giorni dalla stabilizzazione: il prolungamento della degenza, una volta che il paziente si sia stabilizzato, non corrisponde a esigenze di salvaguardia del paziente ma ad esigenze di ansia del medico e talvolta dei familiari del paziente. Questo obiettivo può essere realizzato più facilmente se tutti i medici coinvolti lavorano in team e se l'assistenza ospedaliera trova maggiore raccordi operativi e collaborativi con il territorio (63-65).

\section{INVIO IN UNITÀ DI TERAPIA INTENSIVA}

Quando le condizioni cliniche sono molto gravi si deve ricorrere alla gestione del paziente in Unità di Terapia Intensiva. Le diverse linee-guida sulla CAP considerano un insieme di parametri da valutare per prendere tale decisione: i criteri variano per alcuni dettagli ma sono sostanzialmente omogenei. Il trasferimento in UTI è ritenuto necessario quando vi sia uno stato di grave insufficienza respiratoria o di grave instabilità emodinamica o quando compaiano gravi alterazioni metaboliche, ematiche, renali o grave insufficienza di altri organi. Nella tabella 12 vengono riportati i parametri suggeriti dal gruppo collaborativo europeo ESOCAP: 1'invio in UTI è fortemente indicato quando esista o persista almeno una delle condizioni previste nella tabella (24).

\section{GRAVE INSUFFICIENZA RESPIRATORIA}

- Frequenza respiratoria $>30 \mathrm{resp} / \mathrm{min}$

- $\mathrm{PaO}_{2} / \mathrm{FIO}_{2}<250 \mathrm{mmHg}$ ( $<200 \mathrm{mmHg}$ se $\left.\mathrm{BPCO}\right)$

- Necessità di ventilazione meccanica

- Aumento opacità radiografica $>50 \%$ entro 48 ore

\section{GRAVE INSTABILITÀ EMODINAMICA}

- Pressione arteriosa sistolica $<90 \mathrm{mmHg}$ o diastolica $<60 \mathrm{mmHg}$

- Necessità di farmaci vasoattivi per oltre quattro ore

- Diuresi $<20 \mathrm{ml} / \mathrm{h}$ (in assenza di ipovolemia)

Tabella 12

Criteri di invio in Unità di Terapia Intensiva (UTI) dei pazienti affetti da CAP grave e complicata (Modificatada: ESOCAP, 1998) (24)

\section{CRITERI METABOLICI O EMATOLOGICI}

- Grave acidosi $(\mathrm{pH}<7.30)$

- Grave coagulazione intravascolare disseminata

- Insufficienza renale acuta che richiede dialisi

GRAVE INSUFFICIENZA DI ALTRI ORGANI 


\begin{tabular}{|c|c|c|c|c|c|c|c|c|}
\hline & \multicolumn{2}{|c|}{ Cura a domicilio } & \multicolumn{6}{|c|}{ Ricoverati in ospedale } \\
\hline & \multirow{3}{*}{$\begin{array}{c}\text { Tot. paz } \\
\text { N. }\end{array}$} & \multirow{3}{*}{$\begin{array}{c}\text { Ricoveri } 2^{\circ} \\
\text { tempo } \%\end{array}$} & \multirow{3}{*}{$\begin{array}{c}\text { Tot. paz } \\
\text { N. }\end{array}$} & \multirow{3}{*}{$\begin{array}{l}\text { Inviati in } \\
\text { UTI \% }\end{array}$} & \multicolumn{4}{|c|}{ Durata ricovero } \\
\hline & & & & & Mediana & "Distribu & "zione \% & pazienti \\
\hline & & & & & gg & $<3 \mathrm{gg}$ & $4-7 \mathrm{gg}$ & $>7 \mathrm{gg}$ \\
\hline $\begin{array}{l}\text { Coorte tot. } \\
\text { Classe } \\
\text { rischio } \\
\end{array}$ & 944 & $\overline{77,4}$ & 1.343 & 99,2 & 7 & 13,1 & 37,3 & 499,6 \\
\hline $\mathrm{I}^{\circ}$ & 587 & 5,1 & 185 & 4,3 & 5 & 26,1 & 48,9 & 25,0 \\
\hline $\mathrm{II}^{\circ}$ & 244 & 8,2 & 233 & 4,3 & 6 & 22,1 & 44,2 & 33,8 \\
\hline $\mathrm{III}^{\circ}$ & 72 & 16,7 & 254 & 5,9 & 7 & 13,1 & 41,0 & 45,8 \\
\hline $\mathrm{IV}^{\circ}$ & 40 & 20,0 & 446 & 11,4 & 9 & 5,9 & 31,3 & 62,8 \\
\hline $\mathrm{V}^{\circ}$ & 1 & 0,0 & 255 & 17,3 & 11 & 3,7 & 23,8 & 72,6 \\
\hline $\mathrm{P}$ & & $<0,001$ & & $<0,001$ & $<0,001$ & $<0,001$ & & \\
\hline
\end{tabular}

Tabella 13

Correlazione tra classi di rischio definite secondo l'algoritmo predittivo di Fine e distribuzioni percentuali dei ricoveri in UTI e della durata (giorni) di degenza rilevati nello studio prospettico PORT (Modificatada: Fine MJ et al, 1997) (11)
Come sembra ovvio, i soggetti che si trovano nella necessità di essere trattati in UTI hanno un rischio elevato di morte. La percentuale di soggetti inviati in UTI nel decorso della CAP è direttamente proporzionale al livello di rischio di morte stimato secondo le classi di Fine (11). La tabella 13 evidenzia come tale correlazione sia evidente e statisticamente significativa sia per i soggetti trattati inizialmente a domicilio che, peggiorando, necessitano di un ricovero in seconda istanza, sia per i soggetti ricoverati in ospedale in prima istanza. D'altra parte l'algoritmo di Fine stima il rischio di morte del paziente utilizzando variabili che sono indicative del livello di gravità dell'infezione.

La durata del trattamento in UTI viene, in genere, limitata al minimo necessario, ma varia molto da paziente a paziente ed in un certo numero di casi il paziente deve essere sottoposto a più di un ciclo di terapia intensiva. In definitiva la gravità clinica ed il ricovero in UTI condizionano la durata complessiva del ricovero ospedaliero dei pazienti CAP, come dimostrato nella tabella 13 (11).

\section{CONCLUSIONE}

La gestione efficiente ed eticamente compatibile di un paziente affetto da CAP richiede che il medico sappia prendere una serie successiva di decisioni cruciali che hanno conseguenze rilevanti sull'evoluzione delle condizioni cliniche del paziente e sul bilancio economico del SSN, dell'ospedale, della società ed anche del paziente.

In questo lavoro abbiamo identificato i nodi decisionali cruciali nel percorso asistenziale di un paziente affetto da CAP ed abbiamo cercato di evidenziare alcune relazioni fondamenali tra fattori che determinano le scelte del medico, nonché alcune conseguenze sanitarie delle scelte stesse come appaiono da alcuni lavori pubblicati nella letteratura internazionale.

Il materiale che abbiamo selezionato ed esaminato e le considerazioni che abbiamo posto in discussione sono la piattaforma sulla quale costruirre un modello decisionale per verificare la costo efficacia delle diverse alternative gestionali $\mathrm{e}$, più specificatamente, delle diverse alternative chemioterapiche. Nella seconda parte del lavoro (pubblicata nel prossimo numero) presenteremo tale modello decisionale e verificheremo criticamente l'applicabilità alla situazione italiana.

\section{BIBLIOGRAFIA}

1. Garibaldi RA. Epidemiology od community-acquired respiratory tract infections in adults; incidence, etiology, and impact. Am J Med 1985; 78: 32-37.

2. Dans PE, Charache P, Fahey M, Otter SE. Management of pneumonia in the prospective payment era: a need for more clinician and support service interaction. Arch Intern Med 1984; 144: 1392-1397.

3. La Force FM. Community-acquired lower respiratory tract infections: prevention and cost-control strategies. Am J Med 1985; 78: 52-57. 
4. Graves EJ, Gillum BS. 1994 Summary: National Hospital Discharge Survey. Advance data from vital and health statistics; no 278. Hyattsville, MD: National Center for Health Statistics, 1996.

5. Lave JR, Fine MJ, Sankey SS, Hanusa BH, Weissfeld LA, Kapoor WN. Hospitalized pneumonia: outcomes, treatment patterns, and costs in urban and rural areas. J Gen Intern Med 1996; 11: 415-421.

6. Fine MJ, Hough LJ, Medsger AT, Li YH, Ricci EM, Singer DE, Marrie TJ, Coley CM, Walsh MB, Karpf M, Lahive KC, Kapoor N. Hospital admission decision for patients with community-acquired pneumonia: results from the Penumonia PORT cohort study. Arch Intern Med 1997a; 157: 36-44.

7. Woodhead MA, Macfarlane JT, McCracken JS, Rose DH, Finch RG. Prospective study of the aetiology and outcome of pneumonia in the community. Lancet 1987; 1: 671-674.

8. De Lalla F, Eandi M. Indagine sull'uso di antibiotici nel trattamento delle infezioni delle basse vie respiratorie negli ospedali italiani: considerazioni farmacoeconomiche. Giornale Italiano di Chemioterapia 1998; 45: 5987.

9. McMahon LF Jr, Wolfe RA, tdeschi PJ: Variation in hospital admissions among small areas: a comparison of Maine and Michigan. Med Care 1989; 27: 623-631.

10. Schaberg T, Gialdroni-Grassi G, Huchon G, Leophonte P, Manresa F, Woodhead M. An analysis of decisions by European general practitioners to admit to hospital patients with lower respiratory tract infections. Thorax 1996; 51: 1017-1022.

11. Fine MJ, Auble TE, Yealy DM, Hanusa BH, Weissfeld LA, Singer DE, Coley CM, Marrie TJ, Kapoor WN. A prediction rule to identify low-risk patients with community-acquired pneumonia. N Engl J Med 1997b; 336: 243-250.

12. Coley CM, Li YH, Medsger AR, et al. Preferences for home vs hospital care among low-risk patients with community-acquired pneumonia. Arch Intern Med 1996; 156: 1565-1571.

13. Fine MJ, Smith MA, Carson CA, et al. Prognosis and outcomes of patients with community-acquired pneumonia: a meta-analysis. JAMA 1996; 275: 134-141.

14. Falcone F, Sarno N, Majorano M, Logroscino C. Le polmoniti nell'anziano: epidemiologia. Dati ISTAT del triennio 1989-1991. (in press)

15. Woodhead M, Huchon G, Gialdroni-Grassi G, Leophonte P, Manresa F, Schaberg T. Investigation of lower respiratory tract infection in the community: a European survey. Eur Respir J 1996;

16. Huchon GJ, Gialdroni-Grassi G, Leophonte P, Manresa F, Schaberg T, Woodhead M. Initial antibiotic therapy for lower respiratory tract infection in the community : a European survey. Eur Respir J 1996; 9: 1590-1595.

17. SPILF. Infections des voies respiratoires. Conférence de consensus en thérapeutique anti-infectieuse. Rev Ned Infect $1991 ; 21: 1 \mathrm{~S}-8 \mathrm{~S}$.

18. SEPAR. National recommendation for diagnosis and treatment of community-acquired pneumonia. 1992.

19. American Thoracic Society. Guidelines for the initial management of adults with community-acquierd pneumonia: diagnosis, assesment of severity, and initial antimicrobial therapy. Am Rev Respir Dis 1993, 148: 1418-1426.

20. The British Thoracic Society. Guidelines for the management of community-acquired pneumonia in adults admitted to hospital. Br J Hosp Med 1993; 49: 346-350.

21. Mandell LA, Niederman M. The Canadian Community-Acquired Pneumonia Consensus Conference Group. Antimicrobial treatment of community-acquired pneumonia in adults: a conference report. Can J Infect Dis $1993 ; 4: 25$.

22. Gialdroni-Grassi G, Branchi L. Guidelines for the management of community-acquired pneumonia in adults. Monaldi Arch Chest Dis 1995; 50: 21-27.

23. Bartlett JG, Breiman RF, Mandell LA, File TM. Community-acquired pneumonia in adults: guidelines for management. Clin Infect Dis 1998; 26: 811-838.

24. ESOCAP Study Group. Guidelines for mangement of adult community-acquired lower respiratory tract infections. Eur Respir J 1998; 11: 986-991.

25. Bartlett JM, Mundy LM. Community-acquired pneumonia. N Eng J Med 1995; 333: 1618-1624.

26. Metlay JP, Kapoor WN, Fine MJ.Does this patient have community-acquired pneumonia?: diagnosing pneumonia by history and physical examination. JAMA 1997; 278: 1440-1445.

27. Hasley PB, Albaum MN, Li YH, et al. Do pulmonary radiographic findings at presentation predict mortality in 
patients with community-acquierd pneumonia?. Arch Intern Med 1996; 156: 2206-2212.

28. Spiteri MA, Cook DG, Clarke SW. Reliability of eliciting physical signs in examination of the chest. Lancet 1988; 1: 873-875.

29. Diehr P, Wood RW, Bushyhead J, Kueger L, Wolcott B, Tompkins PK. Prediction of pneumonia in outpatients with acute cough: a statistical approach. J Chronic Dis 1984; 37: 215-225.

30. Singal BM, Hedges JR, Radack KL. Decision rules and clinical prediction of penuomia: evaluation of low-yield criteria. Ann Emerg Med 1989; 18: 13-20.

31. Heckerling PS, Tape TG, Wigton RS et al. Clinical prediction rule for pulmonary infiltrates. Ann Intern Med 1990; 113: 664-670.

32. Gennis P, Gallagher J, Falvo C, Baker S, Than W. Clinical criteria for the detection of pneumonia in adults: guidelines for ordering chest roentgenograms in the emergency department. J Emerg Med 1989; 7: 263-268.

33. Emerman CL, Dawson N, Speroff $\mathrm{T}$ et al., Comparison of physician judgment and decision aids for ordering chest radiographs for pneumonia in outpatients. Ann Emerg Med 1991; 20: 1215-1219.

34. Fine MJ, Smith DN, Singer DE. Hospitalization decision in patients with community-acquired pneumonia: a prospective cohort study. Am J Med 1990a, 89: 713-721.

35. Fine MJ, Orloff JJ, Arisumi D et al. Prognosis of patients hospitalized with community-acquired pneumonia. Am J Med 1990b; 88: 1N-8N.

36. Daley J, Jencks S, Darper D, Lenhart G, Thomas N, Walker J. Predicting hospital-associated mortality for Medicare patients: a method for patients with stroke, pneumonia, acute myocardial infarction, and congestive heart failure. JAMA 1988; 260: 3617-3624.

37. Farr BM, Sloman AJ, Fisch MJ. Predicting death in patients hospitalized for community-acquired pneumonia. Ann Intern Med 1991; 115: 428-436.

38. Marrie TJ, Durant H, Yates L. Community-acquired pneumonia requiring hospitalization: 5-year prospective study. Rev Infect Dis 1989; 11: 586-599.

39. Marrie T, Durant H, Sealy E. Pneumonia: the quality of medical records data. Med Care 1987; $25: 20$.

40. Ortqvist A, Hedlund J, Grillner L et al. Aetiology, outcome and prognostic factors in community-acquired pneumonia requiring hospitalization. Eur Respir J 1990; 3: 1105-1115.

41. Fine MJ, Singer DE, Hanusa BH, Lave JR, Kapoor WN. Validation of a pneumonia prognostic index using the MedisGroups Comparative Hospital Database. Am J Med 1993; 94: 153-159.

42. Eandi M. Farmacoeconomia della terapia antibiotica iniettiva extra-ospedaliera. In: M Eandi et al (Eds), "Terapia Antibiotica Iniettiva Domiciliare (TAID): Clinica, Farmacologia e Farmacoeconomia". Edimes, Pavia, 1998.

43. Macfarlane JT. Treatment of lower respiratory tract infections. Lancet 1987; ii: 1446-1449.

44. Woodhead M. Pneumonia in the elderly. J Antimicrob Chemother 1994; 34 (Suppl. A):85-92.

45. Gleason PP, Kapoor WN, Stone RA, Lave JR, Obrosky DS, Schultz R, Singer DE, Coley CM, Marrie TJ, Fine MJ. Medical outcomes and antimicrobial Costs with the use of the American Thoraci Society Guidelines for outpatients with community-acquired pneumonia. JAMA 1997; 278: 32-39.

46. Paladino JA, Sperry HE, Backes JM et al. Clinical and economic evaluation of oral ciprofloxacin after an abbreviated course of intravenous antibiotics. Am J Med 1991; 91: 462-470.

47. Gentry LO, Rodriguez-Gomez G, Kohler RB, Khan FA, Rytel MW. Parenteral followed by oral ofloxacin for nosocomial and community-acquired pneumonia requiring hospitalization. Am Rev Respir Dis 1992; 145: 3135 .

48. Ehrenkranz NJ, Nerenberg DE, Shultz JM, Slater KC. Intervention to discontinue parenteral antimicrobial therapy in patients hospitalized with pulmonary infections: effect on shortening patient stay. Infect Control Hosp Epidemiol 1992; 13. 21-32.

49. Ramirez JA, Srinath L, Ahkee S, Huang A, Raff MJ. Early switch from intravenous to oral cephalosporins in the treatment of hospitalized patients with community-acquired pneumonia. Arch Intern Med 1995; 155: 12731276.

50. Halm EA, Fine MJ, Marrie TJ, Coley CM, Kapoor WN, Obrosky DS, Singer DE. Time to clinical stability for patients with community-acquired pneumonia: implication for practice guidelines. JAMA 1998; 279: 1452-1457. 
51. Weingarten SR, Riedinger MS, Varis G, et al. Identification of low-risk hospitalized patients with pneumonia. Chest 1994; 105: 1109-1115.

52. Rogers JL, Feinglass J, Martin GJ, et al. Longer hospitalization at veterans administration hospitals than private hospitals. Med Care 1989; 27: 928-936.

53. Burns LA, Wholey DR. The effects of patient, hospital, and physician characteristics on lenght of stay and mortality. Med Care 1991; 29: 225-271.

54. Fine MJ, Singer DE, Phelps AL et al. Differences in lenght of hospital stay in patients with community-acquired pneumonia: a prospective four hospital study. Med Care 1993b; 31: 371-380.

55. Lagoe RA. Community-based analysis of regional differences in hospital stays by diagnosis related group. Inquiry 1986; 23: 183-190.

56. McCormick D, Singer DE, Coley CM et al. Variation in lenght of hospital stay and its relation to medical outcomes in patients with community-acquired pneumonia. J Gen Intern Med 1996

57. McCormick D, Fine MJ, Coley CM, Marrie TJ, Lave JR, Obrosky DS, Kapoor WN, Singer DE. Variation in lenght of hospital stay in patients with community-acquired pneumonia: are shorter stays associated with worese medical outcomes?. Am J Med 1999; 107: 5-12.

58. Feinglass J, Martin GJ, Sen A. The financial effect of physician practice style on hospital resource use. Health Serv Res 1991, 26: 183-205.

59. Horn SD. Sharkey PD, Buckle JM, et al. The relationship between severity of illness and hospital length of stay and mortality. Med Care 1991; 29: 305-317.

60. Stearns SC. Hospital discharge decision, health outcomes and the use of unobserved information on case-mix severity. Health Serv Res 1991; 26: 27-51.

61. Brownell MD, Roos NP. Variation in lenght of stay as a measure of efficiency in Manitoba hospitals. Can Med Assoc J. 1995; 152: 675-682.

62. Weitzman S, Heimer D, Naggan 1 et al., Uncomplicated pneumonia: an evaluation of determinants of lenght of hospitalization. Isr J Med Sci 1983; 19: 591-595.

63. Siegel RE, Halpern NA, Almenoff PL, Lee A, Cashin R, Greene JG. A prospective randomized study of inpatients IV antibiotics for community.acquired pneumonia: the optimal duration of therapy. Chest 1996; 110: $965-971$.

64. Atlas SJ, Benzer TI, Borowsky LH et al. Savely increasing the proportion of patients with community-acquired penumonia treated as outpatients. An interventional trial. Arch Intern Med 1998; 158: 1350-1356.

65. Moher D, Weinberg A, Hanlon R, Runnalls K. Effects of a medical team coordinator on lenght of hospital stay. Can Med Assoc J. 1992; 146: 511-515. 